\title{
EDITORIAL 2020: Journal of Thermal Analysis and Calorimetry
}

\author{
Imre Miklós Szilágyi ${ }^{1}$ - Alfréd Kállay-Menyhárd ${ }^{2} \cdot$ Sophie Korda ${ }^{3}$
}

Published online: 3 December 2019

๑) Akadémiai Kiadó, Budapest, Hungary 2019

\section{Dear Readers,}

2019 was an exciting year in the life of our Journal and we would like to thank all of you for your contributions as Authors, Reviewers, Consultants as well as Associate, Honorary, and Guest Editors. We are grateful to you, because your high-quality work is the guarantee for the success of our Journal.

It is a great news that the impact factor for 2019 was 2.471 , which is ca. $12 \%$ increase compared to the previous year. Although the impact factor for 2018 was already a new record for the Journal, the impact factor for 2019 has surpassed it even considerably more. The value of this impact factor increase is even higher, taking into account that the number of papers published is growing year by year.

Several special issues were published in 2019. We continued our long-term tradition and selected papers presented at international conferences (ICHMT2017, CTAS2017, ICTAES2018, ESTAC12, AICAT2018, XICBRATEC, NATAS2018, CCTA13) were published in JTAC in 2019. As a novelty, a topical special issue on nanofluids (Nanofluid Today) appeared, which was made open access for 2 months, as a courtesy of SpringerNature. This special issue was very popular with nearly 300 papers submitted and more than 100 papers published.

The classification of the manuscripts submitted to our Journal is presented in Fig. 1. We are very happy that the number of manuscript submitted in 2019 is expected to be around 3100 pieces. This is a huge number, and it means

Imre Miklós Szilágyi

imre.szilagyi@mail.bme.hu

1 Department of Inorganic and Analytical Chemistry, Budapest University of Technology and Economics, Szt. Gellért Square 4, Budapest 1111, Hungary

2 Department of Physical Chemistry and Materials Science, Budapest University of Technology and Economics, P.O. Box 92, Budapest 1521, Hungary

3 Akadémiai Kiadó, Budafoki út 187-189, Budapest 1117, Hungary ca. $20 \%$ increase, compared to 2018 . This shows that the journal is becoming even more and more popular in the field of thermal analysis and calorimetry, and thermal science in general. This steep increase, however, is accompanied by new challenges for the future. The most difficult task will be the significant improvement in the average progress time of the submitted manuscripts, but our team is committed to solve this issue in the forthcoming years.

2019 was a very special year; it was the 50th anniversary of the Journal of Thermal Analysis and Calorimetry. We celebrated it with a conference in Budapest, Hungary, where JTAC was founded and where also the first commercial simultaneous TG/DTA device of the world, the famous Derivatograph, was developed.

This was the second edition of our international conference series on thermal analysis and calorimetry, the Journal of Thermal Analysis and Calorimetry Conference (JTACC). During the five decades of publishing, a global community formed around JTAC, and with this conference we wished to create an opportunity, where our editorial board, authors, reviewers, supporters, and all those who were interested in this wonderful field could meet personally.

Accordingly, the 2nd Journal of Thermal Analysis and Calorimetry Conference and 7th V4 (Joint Czech-Hungarian-Polish-Slovakian) Thermoanalytical Conference JTACC+V4 were held in Budapest, Hungary, June 18-21, 2019. Beside tradition, innovation was also present, e.g. electronic poster sessions, conference mobile application, online question and answer part after lectures using sli.do, online participant map, etc. At the conference, we had a special workshop, where we could plan the future of JTAC together with you. The Journal's 50th birthday was also celebrated with a festive gala dinner at the Royal Palace of Gödöllő, featured with JTAC birthday cake, JTAC quiz, Hungarian folkdance show with dance teaching afterwards, and party. The conference was a large success, and there were $30 \%$ more registered (530) participants compared to JTACC 2017, from which around 350 people were on site, making JTACC-V4 2019 one of the largest thermal analysis conferences of the recent decades. 
Fig. 1 Classifications of the manuscripts submitted to our Journal in 2019
JTAC manuscript classifications in 2019

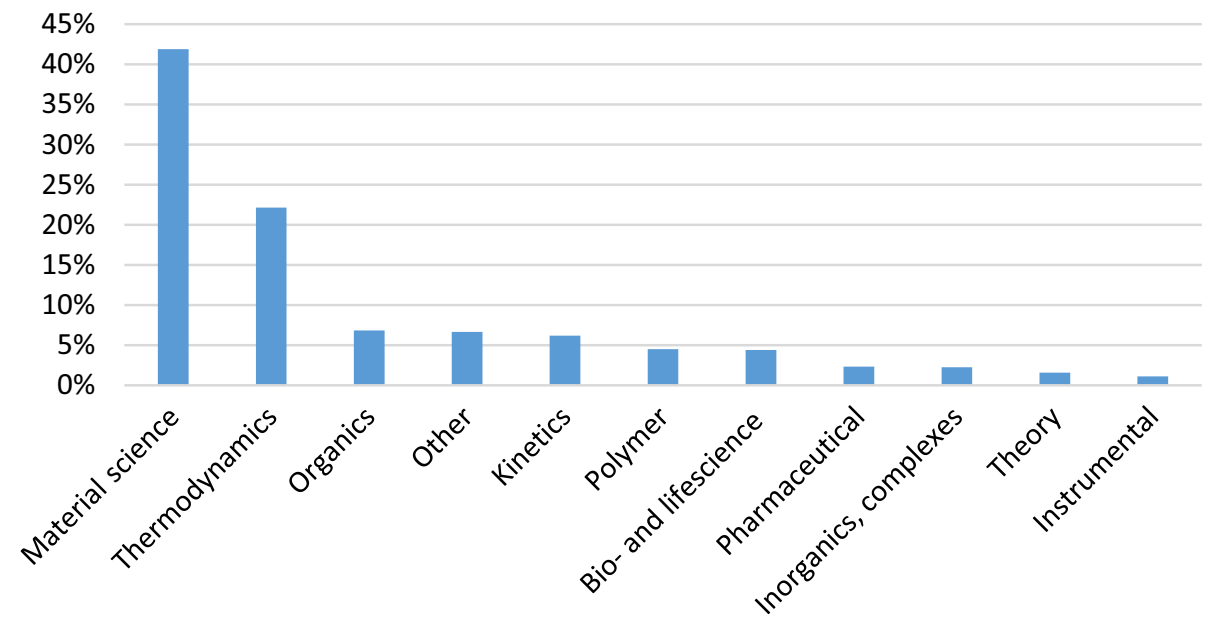

During the conference, our prestigious awards, i.e. the JTAC Scientific Excellence Award, the JTAC Young Scientist Award, and the JTAC Best Reviewer Award, were handed over. The organizing committee assigned the JTAC Scientific Excellence Award to Prof. Barbara Pacewska of the Warsaw University of Technology (Poland) for her outstanding contribution to the development of thermal analysis and calorimetry and for the significant service provided during the years to the community of JTAC. The JTAC Young Scientist Award was given to Dr. Alexander Bannov from the Novosibirsk State Technical University (Russia), who delivered an excellent lecture about the thermal properties of nanostructured carbon materials. The JTAC Best Reviewer Award for the year 2018 was assigned to Prof. Joan Josep Suñol (University of Girona, Spain) for his contribution to improve the quality of JTAC by providing an outstanding number of review reports. Furthermore, by the support of the Ministry of Innovation and Technology (Hungary) JTACC$\mathrm{V} 4$ assigned numerous JTAC and V4 travel grants in order to enable bright and young scientists to attend the JTACC-V4 2019 meeting and thus motivate them to keep a continuing interest and activity in the fields of Thermal Analysis and Calorimetry.

In 2019, several changes were made to increase further the quality of our journal and to please our authors. To reply to the growing popularity of JTAC, we increased the number of papers published, from ca. 850 (2018) to 1050 (2019), which is more than $20 \%$ increase. We introduced new associate editors, especially from the emerging fields of nanofluids, heat transfer, energy, and exergy. Encouraged by the success of the Nanofluid Today special issue, several more topical special issues were initiated in 2019 in the journal.
Finally, we are sad to report that some great researchers have passed away recently, who made groundbreaking steps in thermal analysis and who also contributed significantly to the success of the now 50-year-old JTAC. We are indebted to them to a very large extent. In March 2020, we are going to publish a series of obituaries about these outstanding scientists.

As a closing remark, we would like to wish all our contributors a Very Happy and Successful New Year for 2019.
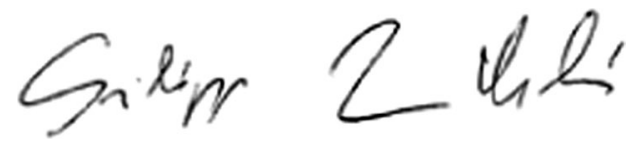

Imre Miklós Szilágyi

Editor-in-Chief
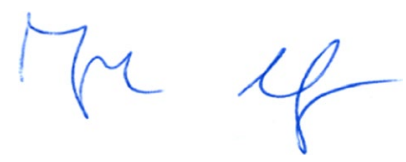

Alfréd Kállay-Menyhárd

Deputy Editor-in-Chief

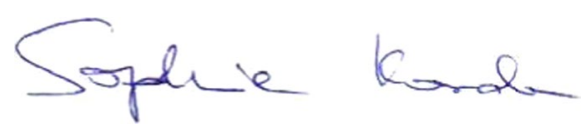

Sophie Korda Head of Editorial Office 


\section{Acknowledgements}

The Editors of the Journal are thankful to the Regional Editors and members of the Associate Editors for their work throughout the past year. We would like to express our special thanks to those referees listed below for their hard work and for the time and expertise they invested to improve the scientific level of the manuscripts. Their work is invaluable in terms of scientific advancement and in providing a constant dynamic forum where all scientists from all over the world can find a professional team helping, educating and inspiring.

Abadeh, A., Iran

Abate, L., Italy

Abatti, L., Brazil

Abbasi, S., Iran

Abbasian Arani, A. A., Iran

Abd El-Ghany, N. A. E.-F., Egypt

Abd Rahim, S., Malaysia

Abdel Gawwad, H., Egypt

Abdelouahed, L., France

Abdulagatov, I., Russian Federation

Abedini-Nassab, R., Iran

Abo Zeid, E. F., Egypt

Achilias, D. S., Greece

Acıkkalp, E., Turkey

Afrand, M., Iran

Afshari, E., Iran

Agarwal, R., India

Agrawal, C., India

Ahmad Hazmi, A. S., Malaysia

Ahmadi Khoshooei, M., Canada

Ahmadi Nadooshan, A., Iran

Ahmadi, M. H., Iran

Ahmed, M. A., Iraq

Akbar, A., Bangladesh

Akbari Fakhrabadi, E., USA

Akbari, M., Iran

Aktas, A., Turkey

Alamo, R., USA

Albu, P. A., Romania

Aleixo, M. L., Brazil

Alekseeva, O. V., Russian Federation

Alex, T. C., India

Alhuyi Nazari, M., Iran

Ali, F., India

Ali, F., Vietnam

Ali, H. M., Saudi Arabia

Ali, M. K. A., Egypt

Ali, S., Italy

Ali, W., India

Allakbakhsh, A., Iran
Allen, N., USA

Alongi, J., Italy

Alonso, A., Spain

Alsabery, A., Malaysia

Al-Sammarraie, A., USA

Alshehri, S. M., Saudi Arabia

Altın, S., Turkey

Alves Rocha, E. P., Brazil

Aly, K. A. N., Egypt

Amani, M., Iran

Amanuel, S., USA

Amaranatha Reddy, D., China

Amin, M. E.-S., Egypt

Amiri Delouei, A., Iran

Amiri, A., Canada

Amiri, A., Malaysia

Amiri, H., Iran

Amiri-Jaghargh, A., Iran

Amitava, B., India

An, W., China

Anand, S., India

Ananth, Dr. V., P., India

Andrade, H. M. C., Brazil

Andrew Pon Abraham, J. D., India

Anghel, E. M., Romania

Angulski da Luz, C., Brazil

Anikina, E. Y., Russian Federation

Anjanapura, R. V., India

Antonovic, V., Lithuania

Ao, W., China

Arai, M., Japan

Arat, H., Turkey

Arena, G., Italy

Armaghani, T., Iran

Arora, C., India

Arsalane, S., Morocco

Arshad, A., UK

Arslan, O., Turkey

Artiaga, R., Spain

Arvizu, M. A., Mexico

Asabina, E. A., Russian Federation

Asadi, A. A., Iran

Assael, M. J., Greece

Ataíde, C. H., Brazil

Atakol, M., Turkey

Atangana, A., South Africa

Atapek, Ş. Hakan, Turkey

Atiqullah, M., Saudi Arabia

Atrooz, O., Jordan

Awad, M. M., Egypt

Aydin, T., Turkey

Aydogdu, V., Turkey

Aytar, A., Turkey 
Ayyasamy, T., India

Azhagurajan, A., India

Aziz, A., Pakistan

Azwadi CheSidik, N., Malaysia

Babaie Rabiee, M., Iran

Baby, R., India

Bachaga, T., Tunisia

Badea, E., Italy

Badea, M. E., Romania

Baghaie, S., Iran

Bahiraei, M., Iran

Bai Wang, S., China

Bai, J., China

Bakic, G., Serbia

Bakri, B., Algeria

Balandin, A., USA

Balasubramanian, D., India

Balkose, D., Turkey

Ballerat, K., France

Baltakys, K., Lithuania

Balu, R., India

Banach, G., Brazil

Baniamerian, Z., Iran

Bannov, A. G., Russian Federation

Barabás, R., Romania

Baranyi, L., Hungary

Bárdos, T., Hungary

Barta Holló, B., Serbia

Bartyzel, A., Poland

Baruah, S. D., India

Barzegar, M., Iran

Barzegarian, R., Iran

Batista, R. M., Brazil

Bauer, J., Poland

Bayareh, M., Iran

Bayer Ozturk, Z., Turkey

Bazri, S., Malaysia

Bejan, A., USA

Belchior Torres, R., Brazil

Belhouchet, H., Algeria

Belina, P., Czech Republic

Bellos, E., Greece

Benchikhi, M., Morocco

Berbenni, V., Italy

Bernardi, L. S., Brazil

Bharadwaj, S., India

Bhattacharyya, A., India

Bhattad, A., India

Bhatti, M. M., China

Bianchi, O., Brazil

Bianco, V., Italy

Bijjana, G. J., India

Bikiaris, D., Greece
Bittencourt, P. R. S., Brazil

Blaine, R., USA

Blanco, I., Italy

Bochenek, D., Poland

Bodzenta, J., Poland

Bohre, A., India

Boldyrev, V., Russian Federation

Boldyreva, E. V., Russian Federation

Boonchom, B., Thailand

Boroica, L., Romania

Borsa, J., Hungary

Borysiak, S., Poland

Bouabdallah, M., Algeria

Boutaous, M., France

Braga, R. M., Brazil

Brahmbhatt, P. K., India

Brandaleze, E., Argentina

Branlund, J., USA

Bright, C., India

Bruni, G., Italy

Bryś, A., Poland

Buczkowski, A., Poland

Budnyak, T. M., Ukraine

Budrugeac, P., Romania

Buriti, J. da S., Brazil

Buschmann, M., Germany

Butt, A., Pakistan

Byczyński, Ł., Poland

Cabaleiro, D., Spain

Cai, X.-f., China

Cairong, Z., China

Carvalho, C. T., Brazil

Castro, R., Portugal

Catana, D., Romania

Cavalheiro, E., Brazil

Cavallaro, G., Italy

Cavallo, D., Italy

Cebe, P., USA

Čelan Korošin, N., Slovenia

Cerc Korošec, R., Slovenia

Cerezci, S., Turkey

Chaipanich, A., Taiwan

Chamkha, A. J., Saudi Arabia

Chandrasekar, M., India

Chang, D.-H., Korea

Chang, J., China

Chang, J.-H., China

Chao, M. J., China

Charmas, B., Poland

Chaudhary, A., India

Chauhan, N., India

Che, D., China

Chen, C., China 
Chen, D., China

Chen, G. Q., China

Chen, J., China

Chen, L., China

Chen, M., China

Chen, R. S., Malaysia

Chen, R., China

Chen, S., China

Chen, S.-P., China

Chen, T., China

Chen, W. T., Taiwan

Chen, X., China

Chen, Y., China

Cheng, H., China

Cheng, J., China

Cheng, Y., China

Chengyun, X., China

Chetehouna, K., France

Chi, J.-H., Taiwan

Chieng, B. W., Malaysia

Chithambaram, V., India

Cholewka, A., Poland

Chou, H.-M., Taiwan

Chougule, S. S., India

Chow, W. S., Malaysia

Chrissafis, K., Greece

Christos, L., Greece

Cibulkova, Z., Slovakia

Ciesinska, W. A., Poland

Colman, T. A. D., Brazil

Cormier, L., Canada

Cosereanu, C., Romania

Crespi, M. S., Brazil

Cristuvao, B., Poland

Crowley, B., USA

Cruz, G., Brazil

Cunha-Filho, M., Brazil

Czégény, Zs., Hungary

Czylkowska, A., Poland

Cseh, Á., Serbia

Cser, F., Australia

Csiszár, E., Hungary

da Silva, E. A., Brazil

da Silva, J. C. G., Brazil

da Silva-Jr, A. A., Brazil

Dagdelen, F., Turkey

Dai, C., China

Dai, P., China

Damodharan, D., India

Das, S., India

Dashti, A., Iran

Dastan, D., USA

Datta, J., Poland
Davalos, J. Z., Spain

Davarnejad, R., Iran

de Guzzi Plepis, A. M., Brazil

de Klerk, W., The Netherlands

de Souza, L. K. C., Brazil

Degroote, E., Spain

Demidaś, A., Poland

Demidova, E., Russian Federation

Deng, T., China

Denkenberger, D. C., USA

Detrich, Á., Hungary

Deymi-Dashtebayaz, M., Iran

Dhanakotti, S., Ethiopia

Dharwadkar, S. R., India

Dias, D. dos S., Brazil

Dindoruk, B., USA

Ding, Q., China

Djellouli, F., Algeria

Doca, N., Romania

Dogan, M., Turkey

Doğan-Sağlamtımur, N., Turkey

Dogonchi, A. S., Iran

Dohnalová, Ž., Czech Republic

Domiri Ganji, D., Iran

Dong, C., China

Dong, G., China

Dong, H., China

Doranehgard, M. H., Canada

Dou, Q., China

Dou, Y., China

Drebushchak, V. A., Russian Federation

Dreizin, E., USA

Drzazga, Z., Poland

Duan, X., Canada

Dubaj, T., Slovakia

Dupont, V., UK

Durmaz, M., Turkey

Duruturk, N., Turkey

Dweck, J., Brazil

E.-Gamal, S. M. A., Egypt

Ebrahimnia-Bajestan, E., Iran

Ehlert, S., Germany

Ehyaei, M. A., Iran

Eisinas, A., Lithuania

Ellahi, R., USA

El-Maghlany, W. M., Egypt

El-Reash, G., Egypt

Elsaid, A. M., Egypt

Emen, F. M., Turkey

Erbaş, K. C., Turkey

Erceg, M., Croatia

Eren, B., Turkey

Esen, M., Turkey 
Esfahani, J. A., Iran

Esfandeh, S., Iran

Esfe, M. H., Iran

Eslami, A., Iran

Estellé, P., France

Fan, C. G., China

Fan, H., China

Farhadi, M., Iran

Farjas, J., Spain

Farshad, S. A., Iran

Farzaneh-Gord, M., Iran

Farzi, A., Iran

Fatoorehchi, H., Iran

Favergeon, L., France

Fayet, G., France

Feng, J., China

Feng, P., China

Feng, Y., China

Feng-Qi, Z., China

Ferencz, A., Hungary

Fernandes Jr., V. J., Brazil

Fierascu, R. C., Romania

Firouzi, M., Iran

Focke, W. W., South Africa

Fonseca, G., Chile

Freitas, V. L. S., Portugal

Frost, R. L., Australia

Fu, X. L., China

Gahleitner, M., Austria

Galysh, V., Ukraine

Gama, R. M. da, Brazil

Gandomkar, A., USA

Ganeeva, Y., Russian Federation

Gao, M., China

Garcia-Sanchez, E., Mexico

Gaur, M. S., India

Gavrichev, K. S., Russian Federation

Ge, H., China

Ge, S., China

Gelfuso, G. M., Brazil

Ghadi, A. Z., Iran

Ghafourian, M., Iran

Ghahremannezhaz, A., USA

Ghalambaz, M., Romania

Ghasemi, A., USA

Gheitaghy, A. M., The Netherlands

Giancola, C., Italy

Gil-Calvo, M., Spain

Goharshadi, E. K., Iran

Goldfarb, J. L., USA

Gomes, E. C. L., Brazil

Gómez Marigliano, A. C., Argentina

Gómez, X., Spain
Gómez-Aguilar, J. F., Mexico

Gong, Z., China

Gonzalez, J. A., Spain

Goodarzi, M., Vietnam

Górniak, A., Poland

Govindarajan, S., India

Gökçe, A., Turkey

Grady, B. P., USA

Greco, A., Italy

Grochwicz, M., Poland

Grubisic-Cabo, F., Croatia

Grząbka-Zasadzińska, A., Poland

$\mathrm{Gu}, \mathrm{X}$., China

Guimarães, D., Brazil

Guirguis, O. W., Egypt

Gultekin, E. E., Turkey

Gun'ko, V., Ukraine

Gunduz, M., Turkey

Gungor, A., Turkey

Guo, F., China

Guo, L., China

Guo, S., China

Guo, W., China

Guo, Y., China

Guo, Z., China

Guzman, L., Chile

Gyarmati, B., Hungary

Hadler, K., UK

Hafiz, M. A., Pakistan

Hajabdollahi Ouderji, Z., Korea

Hakkarainen, T., Finland

Haman, D., Algeria

Han, C., China

Han, F., China

Han, L., China

Hao, J., China

Haribabu, K., India

Hashim, I., Malaysia

Hatanpää, T., Finland

He, F.-A., China

He, L., China

He, Z., China

Hedayat, M., USA

Heikal, M., Saudi Arabia

Hemmat Esfe, M., Iran

Herce, C., Italy

Heris, S. Z., Iran

Hernandez, L., Spain

Hetmańczyk, Ł., Poland

Heydari, A., Iran

Heyhat, M. M., Iran

Hlavička, V., Hungary

Hnilicka, F., Czech Republic 
Hobbs, M., USA

Hofman, T., Poland

Holakooei, P., Iran

Holanda, B. B. da C., Brazil

Holanda, J. N. F., Brazil

Honcova, P., Czech Republic

Hongbo, L., China

Horvat, G., Croatia

Horváth, F., Hungary

Hosoz, M., Turkey

Hosseini, S. E., USA

Hosseini, S. G., Iran

Hosseinirad, E., Iran

Hosseinzadeh, S., Iran

Hou, D., China

Howell, B. A., USA

Hreščak, J., Slovenia

Hristov, J. Y., Bulgaria

Hsiao, K. L., Taiwan

$\mathrm{Hu}, \mathrm{L}$., China

$\mathrm{Hu}$, S., China

Hu, Y., China

Hu, Y.-J., China

Huang, J., China

Huang, W. M., Singapore

Huang, Z., China

Hudson, K., USA

Húlan, T., Slovakia

Huminic, A., Romania

Huminic, G., Romania

Hussein, A. M., Iraq

Hussein, A., Iraq

Ichiyanagi, Y., Japan

Ikeda, H., Japan

Ikonić, B., Serbia

Ilani-Kashkouli, P., USA

Imiela, M., Poland

Imre, A. R., Hungary

Ionashiro, M., Brazil

Ipek, M., Turkey

Irassar, E. F., Argentina

Ishak, A., Malaysia

Ismael, M., Iraq

Iván, B., Hungary

Izadi, M., Iran

Izato, Y.-i., Japan

Jabari Moghadam, A., Iran

Jablonski, M., Poland

Jaćimović, Ž. K., Montenegro

Jafarian, A., Iran

Jafaryar, M., Iran

Jahanian, O., Iran

Jain, A., India
Jaiswar, G., India

Jakić, M., Croatia

Jamaati, J., Iran

Jangili, S., India

Janković, B., Serbia

Janotka, I., Slovakia

Javaherdeh, K., Iran

Jegadheeswaran, S., India

Jena, H., India

Jendrzejczyk-Handzlik, D., Poland

Jeyakumar, N., India

Jha, P. K., India

Ji, J., China

Jia, S., China

Jiang, B., China

Jiang, J., China

Jiang, L., China

Jiao, Y., China

Jiaqiang, E., China

Jin, B., China

Jin, H., China

Jin, X., China

Jing, D., China

Jose, G., India

Jouybari, H. J., Iran

Juchelkova, D., Czech Republic

Judovits, L., USA

Jun, Y., China

Junca, E., Brazil

Kabeel, A. E., Egypt

Kabir, K. B., Bangladesh

Kahani, M., Iran

Kalantar, V., Iran

Kalbasi, R., Iran

Kaletunc, USA

Kalinkin, A. M., Russian Federation

Kaljuvee, T., Estonia

Kamel, M. S., Hungary

Kamseu, E., France

Kandasamy, J., France

Kaptay, Gy., Hungary

Karimi, N., UK

Karimipour, A., Iran

Karolewicz, B., Poland

Karthickeyan, V., India

Kasaeian, A., Iran

Kasprzyk, T., Poland

Katal, R., Austria

Katoh, K., Japan

Kaygili, O., Turkey

Kazemi-Beydokhti, A., Iran

Kefayati, G., Australia

Kelnar, I., Czech Republic 
Keppert, M., Czech Republic

Kerekes, Zs., Hungary

Kesavulu, C. R., Korea

Keshavarz, M. H., Iran

Khabiri, B., Canada

Khaki Jamei, M., Iran

Khalameida, S., Ukraine

Khan, I., Saudi Arabia

Khiari, K., Algeria

Khimeche, K., Algeria

Khoshvaght-Aliabadi, M., Iran

Khosravi-Bizhaem, H., Iran

Kim, J. H., Korea

Kim, S., Korea

Kimura, A., Japan

Kimura, T., Japan

Kirichenko, O., Russian Federation

Kiseleva, D. V., Russian Federation

Klapotke, T. M., Germany

Klebert, Sz., Hungary

Klimm, D., Germany

Kobelnik, M., Brazil

Kocherbitov, V., Sweden

Kodirov, D., Russian Federation

Koga, N., Japan

Kolsi, L., Saudi Arabia

Kong, D., China

Kong, Q., China

Kong, W., China

Konieczny, J. J., Poland

Kopecskó, K., Hungary

Kosar, A., Turkey

Kosinova, M., Russian Federation

Kossoy, A., Russian Federation

Kótai, L., Hungary

Kothari, R., India

Kover, M., Slovakia

Kozlov, A., Russian Federation

Kök, M. V., Turkey

Kök, M., Turkey

Krawczyk, M. B., Poland

Krishna, M. S., India

Krishnamoorthi, T., India

Kristóf, J., Hungary

Król, M., Poland

Krupinska, B., Poland

Ksepko, E., Poland

Kubátová, D., Czech Republic

Kucerik, J., Czech Republic

Kudryavtsev, Y. V., Russian Federation

Kumar, K. G., India

Kumar, P. C., M., India

Kumar, S., India
Kumar, V., India

Kurniawan, R., Korea

Kustov, A., Russian Federation

Kuzielova, E., Slovakia

Künzel, M., Czech Republic

Kwok, Q., Canada

Labisz, K., Poland

Labus, M., Poland

Lacerda, L., Brazil

Lafuente, C., Spain

Lager, D., Austria

Laghzizil, A., Morocco

Lakatos, Á., Hungary

Lalia-Kantouri, M., Greece

Lan, X., China

Larki, M., Malaysia

Larring, Y., Norway

László, K., Hungary

Laureti, S., Italy

Lavasani, A., Iran

Lazzara, G., Italy

Lazzarotto, M., Brazil

Ledeti, I. V., Romania

Lehto, V.-P., Finland

Lei, J., China

Leles, M. I. G., Brazil

Leonelli, C., Italy

Lerchner, J., Germany

Leveneur, S., France

Li, A., China

Li, D., China

Li, G., USA

Li, H., China

Li, J., China

Li, L., China

Li, P., China

Li, Q., China

Li, S., China

Li, Y., China

Li, Z., China

Liao, S., China

Liao, Y. G., China

Liaw, H.-J., Taiwan

Lijie, I., China

Lim, A. R., Korea

Lima, C. R. R. de C., Brazil

Lin, B., China

Lin, C.-P., Taiwan

Lin, Y., China

Liska, M., Slovakia

Lisnyak, V. V., Ukraine

Liu Z.-H., China

Liu, C., China 
Liu, G., China

Liu, H., China

Liu, J., China

Liu, J.-Z., China

Liu, L., China

Liu, L.-L., China

Liu, P., China

Liu, P.-J., China

Liu, Q.-S., China

Liu, R., China

Liu, S., China

Liu, W., China

Liu, X., China

Liu, Y., China

Liu, Y.-W., China

Liu, Z., China

Lobo, B., India

Lochab, B., India

Logvinenko, V. A., Russian Federation

Lomello, M., France

Lomonaco, D., Brazil

Lorenc, J., Poland

Lőrinczy, D., Hungary

Lu, C.-M., China

Lu, P., China

Lu, S., China

Lu, X., China

Lu, Z., China

Lublóy, É. E., Hungary

Luche, J., France

Lujaji, F. C., Tanzania

Luo, Y., China

Lura, P., Switzerland

Luyt, A. S., Qatar

Łyszczek, R., Poland

Lyon, R. E., USA

Lysenko, E., Russian Federation

Ma, H., China

Ma, S., China

Ma, X., China

Ma, Z., China

Maaten, B., Estonia

Mabood, F., Canada

Macan, J., Croatia

Machireddy, G. R., India

Madarász, J., Hungary

Madej, D., Poland

Magdalena, A. G., Brazil

Mahanthesh, B., India

Mahapatra, P. S., India

Maheswaram, M. P. K., USA

Mahian, O., Thailand

Mahmud, S., Canada
Mai, K., China

Majlingová, A., Slovakia

Majoni, S., Botswana

Makhlouf, G. A., Egypt

Makinde, O. D. I., South Africa

Makomaski, G., Poland

Makovicky, E., Denmark

Malaiskiene, J., Lithuania

Malekan, M., Iran

Malghe, Y., India

Maloney, T., Finland

Malucelli, G., Italy

Malyshev, A., Russian Federation

Manasijevic, D., Serbia

Manfredi, L. B., Argentina

Manjubala, I., India

Mao, J., China

Mao, S., USA

Maraden, A. M., Czech Republic

Maraşl1, N., Turkey

Marek, L., Slovakia

Markides, C. N., UK

Marlair, G., France

Marossy, K., Hungary

Martinka, J., Slovakia

Marudova, M., Bulgaria

Masek, A., Poland

Matheswaran, M. M., India

McKenna, G., USA

Mebarek Oudina, F., Algeria

Medved, I., Slovakia

Mei, D., China

Mei, X., China

Melchior, A., Italy

Melnikov, P., Brazil

Meltzer, V., Romania

Mendes, L. C., Brazil

Mendez, A., Spain

Mendoza, O. A., Colombia

Mentado Morales, J., Mexico

Mercuri, F., Italy

Mert, M. S., Turkey

Mesgarpour, M., Iran

Mészáros Szécsényi, K., Serbia

Mészáros, L., Hungary

Michaelides, E., USA

Michałowski, S., Poland

Michel, J. B., Switzerland

Michnik, A., Poland

Mihailov, E., Bulgaria

Mikulová, Z., Czech Republic

Mikuśkiewicz, M., Poland

Minea, A. A., Romania 
Ming, T., China

Mintz, E. A., USA

Mirgolbabaee, H., The Netherlands

Mirparizi, M., Iran

Mishra, D. R., India

Mishra, N., India

Mohamed, M., Egypt

Mohamed, N. A., Egypt

Mohammadi, M., Iran

Mohammadiun, H., Iran

Mohammadiun, M., Iran

Mohammed, H. A., Australia

Mohammed, M., Egypt

Mohan Lal, D., India

Mohan, R., India

Mohanraj, M., India

Mohanty, K., India

Mohd Yunos, N. F. D., Malaysia

Mohebbi, R., Iran

Moita, A., Portugal

Molaeimanesh, G. R., Iran

Molana, M., USA

Montazeri, A., Iran

Monte, M. J. S., Portugal

Montedo, O. R. K., Brazil

Moorthy, B., India

Morais, L. C., Brazil

Morancho, J. M., Spain

Moreno-Piraján, J. C., Colombia

Mothé, C. G., Brazil

Mothe, M., Brazil

Motoc, D. L., Romania

Moudgil, K., USA

Mousavi Ajarostaghi, S. S., Iran

Mousavian, R. T., Iran

Muhammad, T., Pakistan

Muller, A. J., Venezuela

Munteanu, G., Romania

Muraleedharan, K., India

Murali, G., India

Muralidharan, K., India

Muriel, J., Hungary

Murta Valle, M. L., Brazil

Murugapoopathi, S., India

Nadvorny, D., Brazil

Nagappan, b., India

Nagy, B., Hungary

Nagyné Kovács, T., Hungary

Najafi Kani, E., Iran

Nallusamy, N., India

Narasimhan, L., India

Nasir, H., Pakistan

Nath, S. K., India
Nazari M., Iran

Nazir, H., Turkey

Nemes, R., Hungary

Nespoli, A., Italy

Neves Junior, A., Brazil

Nguyen, D. D., Australia

Nguyen, K., Australia

Nicolopolous, S., Belgium

Nie, S., China

Nikolic, J., Serbia

Nishimoto, Y., Bouvet Island

Niyas, H., India

Nocun-Wczelik, W., Poland

Noghrehabadi, A., Iran

Noordin, M. I., Malaysia

Nozela, W. C., Brazil

Nunes, E. N., Brazil

Nunes, R. S., Brazil

Obracaj, D., Poland

Ohata, T., Japan

Ojjela, O., India

Olar, R., Romania

Olewnik-Kruszkowska, E., Poland

Oliveira, P. R., Brazil

Omanovic-Miklicanin, E., Bosnia and Herzegovina

Omidi Kashani, B., Iran

Orozco-Gonzalez, P., Mexico

Ostrowska-Ligeza, E., Poland

Oudadesse, H., France

Ozao, R., Japan

Ozlu, H., Turkey

Ozpozan, N., Turkey

Oztop, H. F., Turkey

Özlü Torun, H., Turkey

Pacewska, B., Poland

Padhye, R., USA

Pal, J., India

Palou, M., Slovakia

Pan, M., China

Pan, W.-P., USA

Pan, Y., China

Panchal, H, India

Panchal, S., Canada

Pandey, India

Panigrahi, B. S., India

Pantoya, M., USA

Panwar, N. L., India

Papageorgiou, G. Z., Greece

Paradeshi, L. B., India

Paramanandam, T., India

Parisi, F., Italy

Pasierb, P., Poland

Pasiut, K., Poland 
Pasman, H., USA

Patel, M. C., India

Pekar, M., Czech Republic

Pelovsky, Y., Bulgaria

Perez-Casas, S., Mexico

Pérez-Maqueda, L., Spain

Pielecha, I., Poland

Pielichowski, K., Poland

Piloto-Rodriguez, R., Cuba

Pinto, R. D. A., Brazil

Pirouzfar, V., Iran

Piz, M., Poland

Plevová, E., Czech Republic

Polyák, P., Hungary

Poncet, S., Canada

Poorfar, A., Iran

Pop, I., Romania

Pop, N., Romania

Popa, A., Romania

Popa, V. T., Romania

Popescu, F., Romania

Popov, A., Latvia

Pouretedal, H. R., Iran

Pourfayaz, F., Iran

Pourmortazavi, S. M., Iran

Pournaderi, P., Iran

Prabhu, S., India

Prakash, C., India

Prasad, M. R. R., India

Predoana, L., Romania

Prica, V.-C., Romania

Priego Quesada, J. I., Spain

Prnová, A., Slovakia

Pruchnik, H., Poland

Puszka, A., Poland

Qasemian, A., Iran

Qian, H., China

Qian, X., China

Qian, X.-M., China

Qian, Y., China

Qiang, W., China

Qiang, X., China

Qiang, Z., USA

Qin, X., China

Qinfu, L., China

Qiu, G., China

Qiu, L., China

Qiu, X., China

Qu, H., China

Rabbi, K. M., USA

Racoviceanu (Babuta) R. M., Romania

Radko, T., Poland

Rafatijo, H., USA
Ragab, A. A., Egypt

Ragula, U. B. R., India

Rahimi, A., Iran

Rahman, A., Qatar

Rahman, M. M., Oman

Rai, U. S., India

Rajeev, R., India

Rajendran, J., India

Ramachandran, T., India

Ramalho, J., France

Raman, V., Saudi Arabia

Ramesan, M. T., India

Ramesh, K., India

Ramrakhyani, M., India

Rapacz-Kmita, A., Poland

Rashad, A. M., Egypt

Rashad, A., Egypt

Rashidi, M. M., China

Rashidi, S., Iran

Rathgeber, C., Germany

Ratlamwala, T. A. H., Pakistan

Ravikumar, T. V. L., India

Razmjoo, R., Spain

Reddy, B. P. A., India

Reddy, G. J., India

Reinmöller, M., Germany

Relkin, P., France

Ren, J., China

Ren, X., China

Restás, Á., Hungary

Ribeiro, C. A., Brazil

Rimez, B., Belgium

Ristić, I. S., Serbia

Rivas, U. R., Spain

Rivera, J., Mexico

Rodrigues, A. C. M., Brazil

Rodrigues, R. V., Brazil

Rodriguez-Anon, J., Spain

Rodríguez-Morales, E., Mexico

Roduit, B., Switzerland

Rogulska, M., Poland

Rokni, H. B., USA

Roldán, W., Chile

Romána, Z., Hungary

Romano, R. C. de O., Brazil

Romero Sanchez, M. D., Spain

Roohi, E., Iran

Rosicka-Kazmarek, J., Poland

Roska, A., Romania

Rostamian, H., Iran

Rostamian, S. H., Iran

Rostamizadeh, M., Iran

Rotaru, A., Romania 
Rotaru, P., Romania

Roy Ghatak, H., India

Roy, S., India

Rutkowski, P. J., Poland

Rybinski, P., Poland

Rycerz, L., Poland

Saad, G., Egypt

Saadeek, Y., Egypt

Sadoughi, M., USA

Sadri, R., Malaysia

Safaei, M. R., Vietnam

Safaei, M., Vietnam

Sahmenko, G., Latvia

Said, Z., United Arab Emirates

Saito, K., Japan

Sajid, M. U., Pakistan

Salahub, D. R., Canada

Salami-Kalajahi, M., Iran

Sałasińska, K., Poland

Salavati-Niasari, M., Iran

Saleem, S., Saudi Arabia

Salifoglou, A., Greece

Salih, A., India

Salimi, F., Iran

Salimpour, M. R., Iran

Samaržija-Jovanović, S., Serbia

Samouillan, V., France

Sankar, S., Korea

Santos, I. M. G. dos, Brazil

Santos, J. C. O., Brazil

Sarafraz, M. m., Iran

Saratovskikh, E., Russian Federation

Saravani, M., USA

Sardarabadi, M., Iran

Sarıkaja, M., Turkey

Sarkar, J., India

Sarkar, S., India

Sarma, A. K., India

Sarra, E., Tunisia

Sathasivam, A., India

Sathyamurthy, R., India

Sato, Y., Japan

Savu, D. I., Romania

Sbirrazzuoli, N., Italy

Scheinherrová, L., Czech Republic

Schiraldi, A., Italy

Schnitzler, E., Brazil

Sebestyén, Z., Hungary

Sedlářová, I., Czech Republic

Sedov, I., Russian Federation

Sekhar, J., India

Selimefendigil, F., Turkey

Sen, A. K., India
Senthil Kumar, R., India

Sesták, J., Czech Republic

Seyednezhad, M., USA

Sfarra, S., Italy

Sha, L., China

Shaaban, E. R., Egypt

Shadloo, M. S., France

Shafee, A., Vietnam

Shafii, M. B., Iran

Shahidian, A., Iran

Shahsavar, A., Iran

Shakeri, F., Iran

Shamlooei, M., Iran

Shanbedi, M., Iran

Shanelova, J., Czech Republic

Sharafeldin, M., Egypt

Sharifpur, M., South Africa

Sharma, R. P., India

Sharma, R., Malaysia

Sharma, S., India

Sharma, V. S., India

Shaw, B. D., USA

Shayanfar, A., Iran

Shehzad, S. A., Pakistan

Sheikhnejad, Y., Portugal

Sheikholeslami, M., Iran

Sheikholeslami, Z., Iran

Shen, W., USA

Shen, Y., China

Sheng, C., China

Sheremet, M., Russian Federation

Shi, Q., China

Shi, Y., China

Shnawa, H. A., Iraq

Short, J., USA

Shoval, S., Israel

Shu, C.-M., Taiwan

Siavashi, M., Iran

Siddiqiui, M. O. R., Pakistan

Sifaoui, H., Algeria

Sikorska, E., Poland

Silva, M. J., Brazil

Silva, R. A. G., Brazil

Šimková, L., Czech Republic

Simon, P., Slovakia

Sinditskii, V. P., Russian Federation

Singh, A. V., India

Singh, A., India

Singh, J., India

Singh, K., India

Singh, P., India

Sitarz, M. T., Poland

Sivakumar, V., Iran 
Skwarek, E., Poland

Smalakys, G., Lithuania

Snopiński, P., Poland

Soares Aragao, C. F., Brazil

Soica, C., Romania

Sojoudi, A., Iran

Sokolowski, J., Canada

Song, J., China

Song, Z., China

Sorrenti, M., Italy

Souza, A. L., Brazil

Souza, R. L., Brazil

Sovizi, M. R., Iran

Spirkova, M., Czech Republic

Srinivasan, K., India

Srivastava, A. P., Ireland

Sroka, Z., Poland

Stanimirova, T., Bulgaria

Stankovic, B., Serbia

Staszczuk, P., Poland

Steiner Petrovic, D., Slovenia

Stevulova, N., Slovakia

Stoch, P., Poland

Stonys, R., Lithuania

Strydom, C., South Africa

Stuart, B., Australia

Subhedar, D., India

Subramanian, T., India

Suceska, M., Croatia

Suchecki, T., Poland

Sudhakar, K., Malaysia

Sugunamma, V., India

Sulcova, P., Czech Republic

Sulochana, C., India

Sulowska, J., Poland

Sun, B., China

Sun, F., China

Sun, J., China

Sun, L., China

Sunden, B., Sweden

Suñol Martinez, J. J., Spain

Suplicz, A., Hungary

Suppiah, D. D., Malaysia

Suresh, S., India

Surianarayanan, M., India

Suseel Jai Krishnan, S., India

Svoboda, R., Czech Republic

Swamy, N. K., India

Szabó-Révész, P., Hungary

Szczygieł, I., Poland

Szeluga, U., Poland

Szostak, E., Poland

Tabak, A., Turkey
Tábi, T., Hungary

Tafarroj, M. M., Iran

Taheri, A., Iran

Takei, T., Japan

Talesh Bahrami, H. R., Iran

Tamizi, E., Iran

Tan, H., China

Tan, Z.-C., China

Tancredi, N., Uruguay

Tang, Y., China

Tao, C., China

Tarhan, M., Turkey

Tarighi, S., Iran

Tarrío-Saavedra, J., Spain

Tascon, J., Spain

Tatraaljai, D., Hungary

Tavares, M. I. B., Brazil

Temuujin, J., Mongolia

Teoh, Y. H., Malaysia

Terekhova, I. V., Russian Federation

Thomas, P., Australia

Thomas, S., USA

Tichapondwa, S. M., South Africa

Tita, B., Romania

Tiwari, A., South Africa

Tiwari, P., India

Toghraie, D., Iran

Tomaszewska-Gras, J., Poland

Tong, J., China

Tongsheng, Z., China

Toplan, H. Ö., Turkey

Toplan, N., Turkey

Torabi, M., Canada

Torabi, M., USA

Torii, S., Japan

Torra, V., Spain

Torres, L. A., Mexico

Trache, D., Algeria

Traskin, V., Russian Federation

Trevisan, M. G., Brazil

Trif, L., Hungary

Trinh, P. V., Vietnam

Tsai, Y.-T., China

Tseng, J.-M., Taiwan

Tsukushi, I., Japan

Tudorachi, N., Romania

Tunç Parlak, T., Turkey

Turkyilmazoglu, M., Turkey

Tutunaru, B., Romania

Tyagi, A. K., India

Tyagi, H., India

Tyagi, S. K., India

Tyagi, V. V., Malaysia 
Tylczyński, Z., Poland

Tylutka, S., Poland

Uddeen, R., India

Ujhelyiová, A., Slovakia

Umavathi, J. C., India

Unal, A., Turkey

Uspenskaja, I. A., Russian Federation

Vaezzadeh, M., Canada

Vafai, K., USA

Vahedi, S. M., Iran

van Ekeren, P. J., The Netherlands

Varganici, C.-D., Romania

Várhegyi, G., Hungary

Vatavuk, J., Brazil

Vecchio Ciprioti, S., Italy

Verevkin, S. P., Germany

Vevkatachalapathy, S., India

Vieru, D., Romania

Vijayakumar, D., India

Vimmrova, A., Czech Republic

Vinukumar, K., India

Vlase, G., Romania

Vlase, T., Romania

Wakif, A., Morocco

Wang, A., China

Wang, B., China

Wang, B.-L., China

Wang, C., China

Wang, C., USA

Wang, C., USA

Wang, D.-Y., Spain

Wang, G., China

Wang, J., Canada

Wang, J., China

Wang, K., China

Wang, N., China

Wang, P.-C., China

Wang, Q., China

Wang, Q., USA

Wang, R., China

Wang, S. F., China

Wang, S., China

Wang, X., China

Wang, X.-D., China

Wang, Y., China

Wang, Y.-W., Taiwan

Wang, Z., China

Wang, Z.-R., China

Waqas, M., Pakistan

Wei, J., China

Wei, K., China

Wei, K., China

Wei, X., China
Wei, Z., China

Wellen, R., Brazil

Wesolowski, M., Poland

Wieczerzak, K., Poland

Wilińska, I., Poland

Wilson, M., UK

Wirawan, R., Indonesia

Wójtowicz, D., Poland

Woodfied, P., Australia

Worzakowska, M., Poland

Wszelaka-Rylik, M., Poland

Wu, F.-g., China

$\mathrm{Wu}$, J., China

Wu, S., China

$\mathrm{Wu}$, S.-H., Taiwan

$\mathrm{Wu}$, Y., China

Wyrzykowski, D., Poland

Wysokowski, M., Poland

Xi, J., China

Xia, W., China

Xianfeng, C., China

Xiao, B., China

Xiao, K., USA

Xiao, L., China

Xiao, Y., China

Xiao, Z., China

Xie, G., China

Xie, H., China

Xie, N., China

Xie, Q., China

Xin, H., China

Xing, X., China

Xu, H., China

Xu, K., China

Xu, Q., China

$\mathrm{Xu}, \mathrm{T}$., China

Xu, T., USA

Xu, Y.-L., China

Yan, B., China

Yan, D., China

Yan, L., China

Yan, P., China

Yan, Q.-L., China

Yan, S., China

Yan, W.-M., Taiwan

Yang, B., China

Yang, F., USA

Yang, L., China

Yang, X.-J., China

Yang, Z., China

Yao, Y., Taiwan

Ye, L., China

Ye, W.-B., China 
Ye, Z., USA

Yi, J., China

Yildiz, K., Turkey

Yilmaz, V. T., Turkey

Yousef, M. S., Egypt

Yousefi, B., Canada

Yousefi, F., Iran

Yousefi, S. H., USA

Yu, Q., China

Yu, Z. W., China

Yuan, B., China

Yuan, Y., China

Yunjun, L., China

Yurdakoc, K., Turkey

Yurddaş, A., Turkey

Zaharescu, M. M., Romania

Zaharescu, T., Romania

Zalga, A., Lithuania

Zamali, H., Tunisia

Zayed, M. A., Egypt

Zecheru, T., Romania

Zeinali Heris, S., Iran

Zelenina, L. N., Russian Federation

Zeman, S., Czech Republic

Zemenová, P., Czech Republic

Zeng, J.-L., China

Zhan, J.-H., China

Zhang, C., China

Zhang, D., China

Zhang, F., China

Zhang, G., China

Zhang, G.-Z., China

Zhang, H., China

Zhang, J. W., China

Zhang, J., Denmark
Zhang, P., China

Zhang, Q., USA

Zhang, S., China

Zhang, W., China

Zhang, X., USA

Zhang, Y., China

Zhang, Y.-F., China

Zhang, Z., China

Zhang, Z., USA

Zhangfeng, Z., China

Zhao, B., China

Zhao, C.-w., China

Zhao, F.-q., China

Zhao, J.-Y., China

Zhao, M., China

Zhao, N., China

Zhao, X., China

Zhao, Y., China

Zheng, C., Sweden

Zheng, J. Y., China

Zhixiang, X., China

Zhong, D., China

Zhong, W., China

Zhou, K., China

Zhou, Q., China

Zhou, W., China

Zhou, X., UK

Zhu, F., China

Zhu, M., Australia

Zhu, M., China

Zhu, M., USA

Publisher's Note Springer Nature remains neutral with regard to jurisdictional claims in published maps and institutional affiliations. 